\title{
Efeitos do "feedback" autocontrolado na aprendizagem do lançamento da bola da ginástica rítmica
}

CDD. 20.ed. 152.3

796.405

\author{
Anielle LEMOS* \\ Suzete CHIVIACOWSKY ${ }^{*}$ \\ Luciana Toaldo Gentilini ÁVILA* \\ Ricardo DREWS*
}

\section{Resumo}

0 objetivo do presente estudo foi investigar os efeitos da frequência autocontrolada de "feedback" na aprendizagem do lançamento da bola da ginástica rítmica. A amostra foi constituída de 24 crianças, de sete a 10 anos de idade, distribuídas em dois grupos em relação aos diferentes tipos de condições de "feedback": autocontrolado e externamente controlado. 0 estudo foi composto de uma fase de prática e um teste de retenção. Os resultados de um questionário revelaram que a maioria dos participantes do grupo autocontrolado solicitou "feedback" principalmente após as boas tentativas de prática, enquanto os participantes do grupo "yoked" indicaram ter recebido "feedback" nas tentativas em que o desejavam. Nenhuma diferença foi encontrada em relação ao desempenho ou aprendizagem. Conclui-se que a frequência autocontrolada de "feedback" é tão eficiente quanto a frequência externamente controlada na aprendizagem da habilidade motora específica lançamento do aparelho bola em crianças desta faixa etária.

PalaVRaS-Chave: Aprendizagem motora; Aprendizagem autocontrolada; Habilidades esportivas; Crianças.

\section{Introdução}

Muitos fatores que afetam a aprendizagem motora estão presentes e precisam ser organizados durante o processo de aprendizagem de habilidades motoras específicas dos esportes. Exemplos podem ser os relacionados à instrução a ser fornecida, à organização da prática, ao fornecimento de "feedback" e também ao autocontrole ou não, de algum destes fatores, por parte do aprendiz ${ }^{1}$.

A aprendizagem autocontrolada é caracterizada por uma atuação mais ativa dos aprendizes no decorrer do seu processo de aprendizagem ${ }^{2}$, considerando que lhes é fornecida a possibilidade de tomar decisões referentes a algum aspecto importante da prática. Resultados recentes de estudos investigando esta variável vêm demonstrando os seus benefícios sobre a aprendizagem motora em diversas situações de prática, principalmente em adultos. Por exemplo, KeEtch e $\mathrm{LeE}^{3}$ e Wu e MAgill ${ }^{4}$ examinaram o uso de estratégias de organização da prática e os resultados demonstraram que o grupo que pode escolher a ordem de prática das diferentes tarefas (grupo autocontrolado) obteve benefícios de aprendizagem em relação aos grupos sem chance de escolha. Já os estudos de Wrisberg e Pein ${ }^{5}$ e Wulf et al. ${ }^{6}$ investigaram a efetividade da condição de prática autocontrolada na escolha de quando receber demonstração de um modelo habilidoso durante a prática. Os resultados mostraram superioridade do grupo autocontrolado em relação aos demais grupos de prática. Foram também encontradas vantagens no aprendizado de tarefas motoras com o uso autocontrolado de ajuda física ${ }^{7-9}$, assim como na escolha do número de tentativas a serem realizadas em uma sessão de prática ${ }^{10}$.

Especificamente com relação a variável frequência de "feedback", um dos primeiros estudos a abordar os efeitos da aprendizagem autocontrolada na aprendizagem motora e a encontrar vantagens da utilização desta variável foi o estudo de JANELLE et al. ${ }^{11}$. Posteriormente, ChIVIACOWSKY e WULF ${ }^{12}$ também encontraram resultados positivos da utilização do "feedback" autocontrolado na aprendizagem motora 
de adultos e foram além, aplicando um questionário aos participantes com a intenção de verificar quando/porquê os mesmos receberam (grupo "self") ou prefeririam receber (grupo "yoked") informaçōes de "feedback": após boas ou más tentativas de prática. Os resultados demonstraram que os participantes do grupo "self" escolheram receber "feedback" após as boas tentativas de prática, enquanto os participantes do grupo "yoked", embora também preferissem receber "feedback" após as tentativas mais eficientes, ou seja, com menor valor de erro, responderam não tê-lo recebido nas tentativas em que o desejavam. Os estudos de Patterson e Carter ${ }^{13}$ e Patterson et al. ${ }^{14}$ replicaram os resultados do questionário encontrados por CHIVIACOWSKY e WULF ${ }^{12}$.

Entretanto, ainda são poucos os estudos encontrados na literatura investigando os efeitos do "feedback" autocontrolado na aprendizagem motora de crianças e os resultados existentes apresentam-se contraditórios. Por exemplo, enquanto um estudo ${ }^{15}$ utilizando uma tarefa com demanda de controle espacial de arremessar saquinhos de feijão em um alvo demonstrou que os benefícios deste tipo de

\section{Método}

\section{Amostra}

A amostra foi constituída de 24 crianças do sexo feminino, na faixa etária de sete a 10 anos de idade (grupo $\mathrm{AC}: \mathrm{M}=8,83 ; \mathrm{SD}=1,19$; grupo "yoked": $\mathrm{M}=8,75, \mathrm{SD}=1,13)$, participantes de um projeto do Serviço Social da Indústria (SESI) na cidade de Pelotas - RS. Os participantes foram distribuídos de forma aleatória, em dois grupos de 12 sujeitos, de acordo com os diferentes tipos de informação de "feedback". Antes da realização da coleta de dados, o projeto do estudo foi submetido ao Comitê de Ética em Pesquisa da Universidade Federal de Pelotas, sendo aprovado com o protocolo de número 033/2011. As crianças participaram como voluntárias e não possuíam conhecimento sobre o objetivo do experimento. A participação das mesmas foi concedida após assinatura do termo de consentimento livre e esclarecido pelos pais ou responsáveis.

\section{Tarefa e equipamento}

O aparelho utilizado foi uma bola oficial da GR, feita com material de borracha, com tamanho de 18 "feedback" também podem se estender a crianças de 10 anos de idade, outro estudo ${ }^{16}$ utilizando uma tarefa temporal sequencial, de pressionar teclas do computador, não encontrou vantagens para o grupo com chance de escolha em relação a um grupo "yoked", na mesma faixa etária. Pode-se inferir, desta forma, que os efeitos positivos do "feedback" autocontrolado podem depender do tipo ou da complexidade da habilidade motora a ser aprendida, já que tais aspectos podem determinar o grau de dificuldade em que resultados mais ou menos eficientes podem ser discriminados pelos aprendizes.

O objetivo do presente estudo foi comparar os efeitos da frequência autocontrolada de "feedback" na aprendizagem do lançamento do aparelho bola, habilidade motora específica da ginástica rítmica (GR), em crianças. Considerando os resultados de estudos prévios, em adultos e crianças, de forma geral, espera-se que os participantes que recebam um arranjo autocontrolado de fornecimento de "feedback" apresentem aprendizagem mais efetiva do que os participantes que não tenham a chance de autocontrolar esta variável.

$\mathrm{cm}$ de diâmetro e peso de $400 \mathrm{~g}$. O lançamento da bola se caracteriza por quatro fases, as quais podem acontecer com ou sem deslocamento do corpo: fase preparatória, onde o aprendiz posiciona a bola na mão ou outra parte do corpo para o lançamento; o lançamento em si, que inicia no momento da realização de um movimento pendular com os braços; fase de voo, que ocorre no momento em que se perde o contato com a bola; e a recepção, que é a fase de aterrissagem da bola sobre a mão ou outra parte do corpo do sujeito ${ }^{17}$. A tarefa do estudo consistiu em realizar um grande lançamento simples da bola, com uma das mãos, com o objetivo de atingir o dobro da altura do sujeito, como estipula o código de pontuação da Federação Internacional de Ginástica (FIG) ${ }^{18}$. Além disto, na fase de voo do aparelho, o participante deveria realizar um deslocamento de três passos em trajetória retilínea para frente e, em seguida, o aparelho deveria ser recepcionado com a mesma mão. Todos os lançamentos foram realizados com a mão dominante de cada sujeito. Foi também aplicado um questionário ao final da fase de aquisição, adaptado do estudo de ChiviacowsKY e WULF ${ }^{12}$. 


\section{Procedimentos}

De acordo com o delineamento experimental, os participantes foram distribuídos de forma aleatória em dois grupos com igual número de participantes: grupo que recebeu frequência de "feedback" autocontrolado (AC) e grupo que recebeu frequência de "feedback" externamente controlada ("Yoked"). Enquanto o grupo AC tinha a chance de solicitar "feedback" sempre que achasse necessário, sem controle do experimentador, o grupo "Yoked" recebia esta informação de forma equiparada, sujeito a sujeito, com o grupo AC, de forma que o número de "feedbacks" solicitados, assim como o espaçamento entre solicitações, foram os mesmos para ambos os grupo.

O experimento constou de uma fase de prática com 60 tentativas e um teste de retenção, realizado cerca de 24 horas após a prática, composto por 10 tentativas da tarefa, sem fornecimento de "feedback". $\mathrm{Na}$ fase de prática os participantes do grupo AC foram informados que controlariam a quantidade de "feedbacks" que gostariam de receber durante a prática, assim como o momento de solicitação dos mesmos. Já os participantes do grupo "Yoked" receberam a instrução de que às vezes receberiam a informação de "feedback" do experimentador e às vezes não, mas que todas as tentativas eram importantes e seriam analisadas posteriormente. $\mathrm{O}$ questionário foi aplicado ao final da fase de prática.
Todas as fases do estudo foram realizadas em um ginásio poliesportivo, utilizando critérios para a marcação da pontuação da habilidade. A avaliação de cada tentativa da tarefa, para fins de análise estatística, foi dividida em três aspectos (lançamento, recepção e deslocamento), as quais receberam pontuaçōes específicas. Para o lançamento, 10 pontos foram fornecidos aos lançamentos considerados eficientes (altura correta), 5 pontos para os regulares (altura média) e 0 pontos para os ineficientes (altura insuficiente). Na recepção, 10 pontos foram dados às recepções consideradas eficientes (com apenas uma mão), 5 pontos para as regulares (com as duas mãos ou a bola tocando no antebraço) e 0 pontos para as ineficientes (queda da bola). E, por último, para o deslocamento, 10 pontos foram dados aos deslocamentos eficientes (três passos), 5 pontos para os regulares (não completa o deslocamento) e 0 pontos para os ineficientes (sem deslocamento). A fim de compor o escore de pontuação por tentativa, foram somados os escores de cada fase da habilidade.

O "feedback" foi fornecido de forma prescritiva aos participantes, com o experimentador utilizando apenas uma das oito informaçōes que se encontram listadas no QUADRO 1, após as tentativas solicitadas pelos participantes do grupo "self" ou, de forma equiparada ao grupo "self", para os participantes do grupo "Yoked". O experimentador escolhia o "feedback" de acordo com o aspecto da performance que refletia a maior necessidade de melhora na respectiva tentativa.

QUADRO 1 - Informações de "feedback" utilizadas para ambos os grupos.

\begin{tabular}{|c|c|}
\hline & Informaçóes de "Feedback" \\
\hline \multirow{4}{*}{ Lançamento } & 1. Lançar mais alto \\
\hline & 2. Lançar mais para frente \\
\hline & 3. Lançar mais baixo \\
\hline & 4. Lançar mais perto \\
\hline \multirow{4}{*}{ Deslocamento } & 5. Caminhar mais \\
\hline & (quando os participantes realizavam menos de três passos) \\
\hline & 6. Caminhar menos \\
\hline & (quando os participantes realizavam mais de três passos) \\
\hline \multirow{2}{*}{ Recepção } & 7. Receber com uma mão só \\
\hline & 8. Não tocar a bola no antebraço \\
\hline
\end{tabular}

\section{Análise dos dados}

$\mathrm{Na}$ análise dos resultados, as curvas de desempenho foram traçadas em função dos blocos de tentativas, tendo como medida da variável dependente a média da soma dos escores atribuídos em cada tentativa para as fases de lançamento, recepção e deslocamento. Para a fase de prática foram realizadas comparações das médias através de uma Análise de variância (ANOVA) sendo 2 (grupo: AC versus "Yoked") x 6 (blocos 
de 10 tentativas), com medidas repetidas no último fator. Já a fase de retenção constou de uma ANOVA "one-way", com apenas um bloco de 10 tentativas.

A fim de determinar se os sujeitos dos diferentes grupos solicitaram/receberam "feedback" principalmente após as boas ou as más tentativas de prática, calculou-se

\section{Resultados}

\section{Prática}

A média de solicitação de "feedbacks" pelos sujeitos do grupo AC foi de 10,2 (DP = 4,64), o que corresponde a uma frequência média de $17 \%$ de "feedback", durante as tentativas de prática. Com relação ao desempenho, pode-se observar, por meio do gráfico (FIGURA 1, bloco $1 \mathrm{a}$ bloco a média de pontuaçôes na tarefa nas tentativas com e sem "feedback" e realizou-se uma ANOVA "twoway", 2 (grupo: AC x "Yoked") x 2 (tipo de tentativa: com "feedback" versus sem "feedback"), com medidas repetidas no último fator. Os dados foram analisados através do programa estatístico SPSS 13.0.

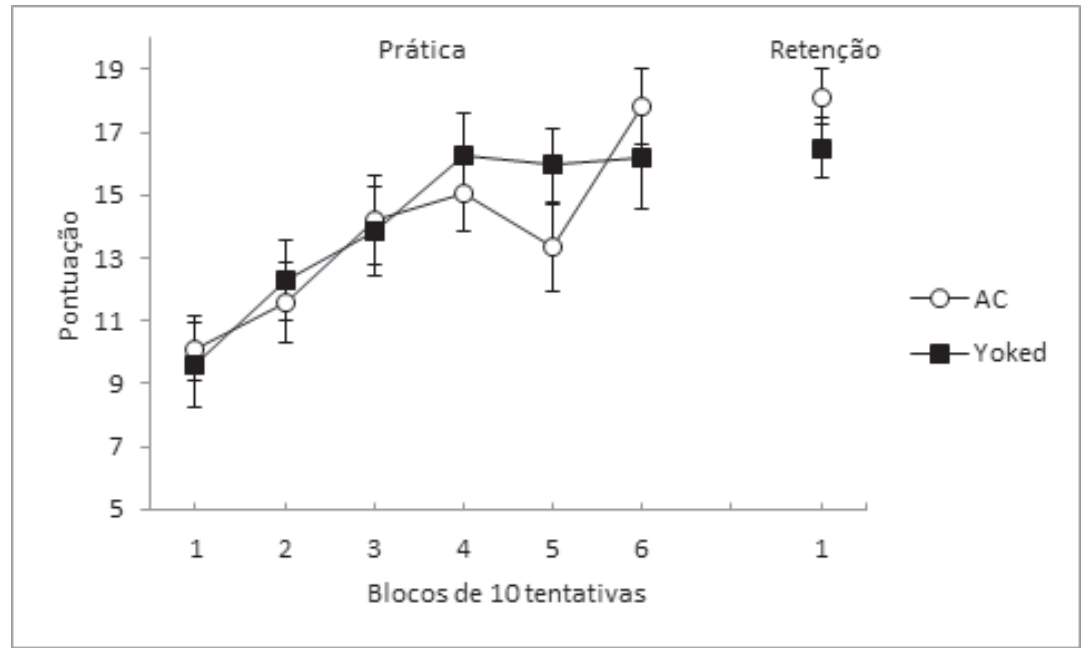

FIGURA 1 - Médias dos resultados dos grupos autocontrolado (AC) e externamente controlado ("Yoked") nas fases de prática e retenção.

\section{Retenção}

$\mathrm{Na}$ fase de retenção, apesar do grupo AC ter alcançado pontuação superior à do grupo "Yoked", a ANOVA "one-way" não mostrou diferença significativa entre os grupos, $\mathrm{F}(1 ; 23)=1,57, \mathrm{p}>0,05$.
6 da fase de prática), que ambos os grupos tiveram um processo de aprendizagem semelhante nesta fase, melhorando o seu desempenho ao longo das tentativas. A ANOVA revelou diferença significativa entre os blocos $\mathrm{F}(5 ; 110)=21,19, \mathrm{p}<0,01, \eta^{2}=$ 0,49 ; mas não entre os grupos $\mathrm{F}(1,22)<1$, ou na interação entre blocos e grupos $\mathrm{F}(5,110)=1,77$, $\mathrm{p}>0,05$. 
QUADRO 2 - Questões e respostas dos sujeitos do grupo autocontrolado (AC) e do grupo externamente controlado ("Yoked").

\begin{tabular}{|lc|}
\hline Grupo & Número de respostas \\
\hline Autocontrolado & 9 \\
1.Quando/ por que você pediu feedback? & 2 \\
a ( ) na maioria das vezes após realizar uma boa tentativa & 1 \\
b ( ) na maioria das vezes após realizar uma má tentativa & 10 \\
c ( ) nenhuma das anteriores. Especifique_ & 2 \\
\hline Yoked & 10 \\
1.Você acha que recebeu informação de feedback após as tentativas em que precisava? & \\
a ( ) Sim & \\
b ( ) Não & 0 \\
2.Se a resposta foi Não: & 2 \\
Você teria preferido recebê-lo após tentativas específicas como: & 0 \\
a ( ) Após tentativas boas? & \\
b ( ) Após tentativas ruins? & \\
c ( ) Nenhuma das anteriores. Especifique_ & \\
\hline
\end{tabular}

\section{"Feedback" após boas versus más tentativas}

A fim de determinar se os participantes solicitaram/receberam "feedback" principalmente após as tentativas mais eficientes de prática foi realizado o cálculo das médias de pontuações das tentativas com e sem "feedback" para ambos os grupos. Nos resultados encontrados, conforme mostra o gráfico da FIGURA 2, pode-se observar maior pontuação média nas tentativas em que o "feedback" foi solicitado do que nas tentativas sem "feedback" para o grupo AC. Já no grupo "Yoked", os resultados mostraram que não houve diferença na pontuação média entre tentativas com e sem "feedback".

Foram verificadas diferenças entre o tipo de tentativa, $\mathrm{F}(1,22)=13,82, \mathrm{p}<0,01, \eta^{2}=0,38$ com valores de pontuação significativamente superiores nas tentativas em que os sujeitos receberam "feedback" em comparação com as tentativas sem "feedback", e na interação entre grupos e tipo de tentativa, $\mathrm{F}(1,22)=12,51$, $\mathrm{p}<$ $0,01, \eta^{2}=0,36$. Tais resultados indicam que os sujeitos do grupo AC receberam mais "feedback" após as boas tentativas de prática do que o grupo "Yoked".

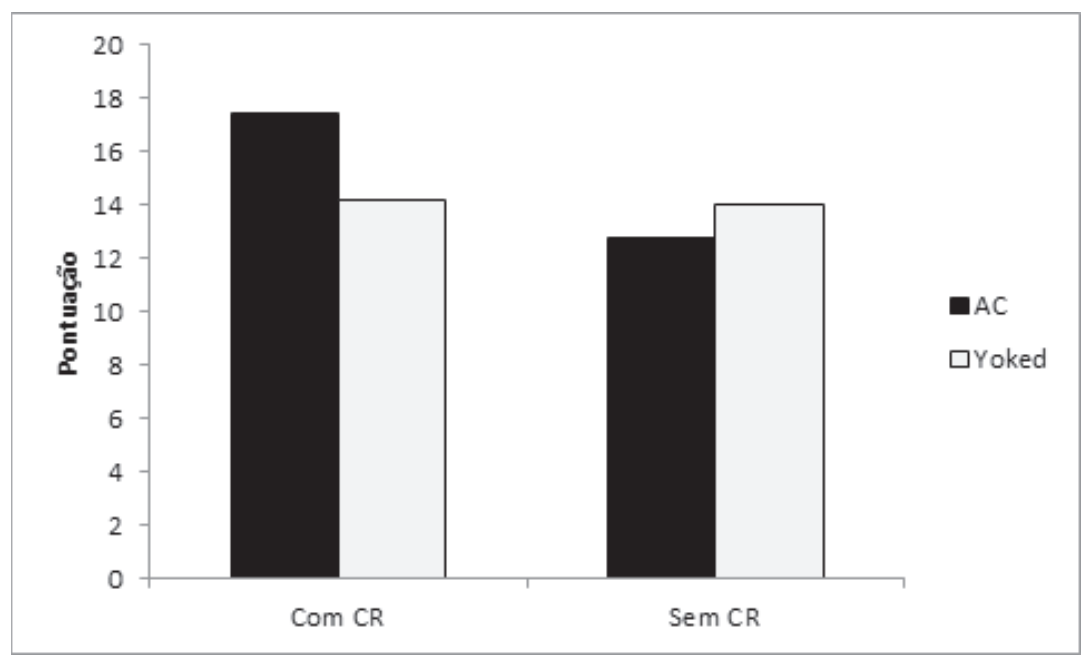

FIGURA 2 - Pontuação da tentativas com CR e sem CR para os diferentes grupos. 


\section{Discussão}

O presente estudo buscou investigar qual a forma mais eficaz de fornecimento de "feedback", autocontrolado ou externamente controlado ("yoked"), para a aprendizagem de uma habilidade motora com o aparelho bola, da GR, em crianças. Os resultados mostraram que ambos os grupos melhoraram o seu desempenho de forma bastante similar durante a prática, enquanto o grupo AC demonstrou maior pontuação do que o grupo "Yoked" na fase de retenção. Entretanto, não foram encontradas diferenças significativas entre os grupos em nenhuma fase do experimento, o que demonstra aprendizagem similar para participantes que praticaram esta tarefa com frequência de "feedback" autocontrolada ou externamente controlada.

Os benefícios da prática autocontrolada têm sido explicados através de diferentes proposiçōes teóricas. Por exemplo, tem sido sugerido que o envolvimento mais ativo do aprendiz, causado pela prática autocontrolada, pode promover um processamento mais profundo de informações (resultando em maior probabilidade das informaçôes alvo serem lembradas) ${ }^{19-21}$, encorajar o uso de estratégias autoreguladas (as quais envolvem um maior nível de autopercepção por parte dos aprendizes durante a busca por informaçôes) $)^{22}$, ou, de forma geral, ser mais motivante ${ }^{23-24}$ do que arranjos de prática onde o aprendiz não exerce controle sobre o aspecto estudado, embora testes empíricos a fim de testar estas hipóteses ainda sejam limitados na literatura até o presente momento.

Alguns estudos específicos da área da aprendizagem motora têm demonstrado, principalmente através de questionários e/ou análise das tentativas de prática, que fatores motivacionais podem ser os responsáveis pelos benefícios do "feedback" autocontrolado ${ }^{12-15,25}$. Os resultados destes estudos têm indicado que a preferência e a possibilidade de receber "feedback" após tentativas eficientes de prática, parecem exercer um papel importante neste processo. Resultados subsequentes de estudos, utilizando "feedback" externamente controlado têm dado suporte a esta proposição, demonstrando que participantes que recebem "feedback" principalmente após as boas tentativas, apresentam maior aprendizagem do que participantes que recebem "feedback" após as más tentativas de prática ${ }^{26-29}$.

Os resultados do presente estudo diferem dos encontrados no estudo de CHIVIACOWsky et al..$^{15}$, com crianças de 10 anos de idade, o qual confirmou os benefícios do "feedback" autocontrolado em relação ao externamente controlado ("yoked") na aprendizagem de uma tarefa de arremessar saquinhos de feijão em um alvo. Por outro lado, os resultados são similares aos observados no estudo de CHIVIACOWSKY et al. ${ }^{16}$, no qual foi utilizada uma tarefa sequencial de pressionar teclas do computador, onde o grupo que praticou com "feedback" autocontrolado também não apresentou aprendizagem superior ao grupo com frequência de "feedback" equiparada ("yoked").

Ao observar-se a discrepância dos resultados dos estudos citados, seria importante considerar os aspectos referentes à preferência ou não dos aprendizes em solicitar/receber "feedback" principalmente após boas tentativas de prática, a capacidade dos mesmos em realmente diferenciar entre tentativas em que foram ou não eficientes, e as possíveis consequências motivacionais e de aprendizagem. Por exemplo, no estudo de Chiviacowsky et al. ${ }^{15}$ os participantes do grupo AC foram capazes de discriminar entre boas e más tentativas, solicitando "feedback" principalmente após as tentativas mais eficientes de prática. No entanto, no estudo de CHIVIACOWsKy et al. ${ }^{16}$, os participantes do grupo $\mathrm{AC}$ não foram eficientes em discriminar entre boas e más tentativas ao solicitar "feedback", apesar da preferência, reportada no questionário, por receber "feedback" após as tentativas eficientes. Tais fatos podem ser responsáveis pela diferença entre os resultados de aprendizagem destes estudos. No presente estudo, a maioria dos participantes do grupo AC preferiram e foram capazes de solicitar "feedback" após as tentativas mais eficientes de prática. Entretanto, observou-se que a maioria dos sujeitos do grupo "yoked" reportaram ter recebido "feedback" exatamente nas tentativas em que o desejavam, igualando-os aos participantes do grupo AC em relação a este aspecto. Se grande parte dos efeitos da prática autocontrolada podem ser causados por fatores motivacionais relacionados ao momento de recebimento do "feedback", de acordo com as preferências e necessidades dos aprendizes ${ }^{12,25}$, pode-se inferir uma igualdade entre os grupos em relação a este aspecto no presente estudo, resultando em similar aprendizagem da tarefa.

Através dos resultados do presente estudo concluise que a frequência autocontrolada de "feedback"é tão eficiente quanto a frequência externamente controlada na aprendizagem da habilidade motora específica lançamento do aparelho bola, da GR, em crianças de sete a 10 anos de idade. Sugere-se novos estudos que verifiquem mais diretamente, além dos 
efeitos de aprendizagem em outras habilidades esportivas, as estratégias utilizadas pelas crianças durante a solicitação de "feedback", assim como os efeitos da prática autocontrolada na sua motivação intrínseca.

\begin{abstract}
Effects of self-controlled feedback on the learning of ball throwing in rhythmic gymnastics

The aim of this study was to investigate the effects of self-controlled frequency of feedback on the learning of ball throwing in rhythmic gymnastics. 24 children, ranging from 7 to 10 -years old participated in the study, divided into two groups in relation to the different feedback conditions: self-controlled and externally controlled (yoked). The study consisted of a practice phase and a retention test. The results of a questionnaire showed that the majority of participants of the self-control group requested feedback mainly after good trials, while participants of the yoked group indicated that they had received feedback on the trials they desired. No differences were found regarding performance or learning. We concluded that self-controlled feedback frequencies are as effective as externally controlled feedback frequencies for the learning of rhythmic gymnastics ball throwing in children.
\end{abstract}

KEY WORDS: Motor learning; Self-controlled learning; Sport skills; Children.

\title{
Referências
}

1. Schmidt RA, Wrisberg CA. Aprendizagem e performance motora: uma abordagem da aprendizagem baseada no problema. 2a ed. Porto Alegre: Artmed; 2011.

2. Chiviacowsky S. Frequência de conhecimento de resultados na aprendizagem motora: linhas atuais de pesquisa e perspectivas. In: Tani G, editor. Comportamento motor: aprendizagem e desenvolvimento. Rio de Janeiro: Guanabara Koogan; 2005. p.185-207.

3. Keetch K, Lee T. The effect of self-regulated andexperimenter-imposed practice schedules on motor learning for tasks of varying difficulty. Res Q Exerc Sport. 2007;78:476-86.

4. Wu WF, Magill RA. Allowing learners to choose: self-controlled practice schedules for learning multiple movement patterns. Res Q Exerc Sport. 2011;82:449-57.

5. Wrisberg CA, Pein RL. Note on learners control of the frequency of model presentation during skill acquisition. Percept Motor Skills. 1992;94:792-4.

6. Wulf G, Raupach R, Pfeiffer F. Self-controlled observational practice enhances learning. Res Q Exerc Sport. 2005;76:10711.

7. Wulf G, Toole T. Physical assistance devices in complex motor skill learning: benefits of a self-controlled practice schedule. Res Q Exerc Sport. 1999;70:265-72.

8. Hartman JM. Self-controlled use of a perceived physical assistance device during a balancing task. Percept Motor Skills. 2007;104:1005-16.

9. Chiviacowsky S, Wulf G, Lewthwaite R, Campos T. Motor learning benefits of self-controlled practice in persons with Parkinson's disease. Gait Posture. 2012;35:601-5.

10. Post PG, Fairbrother JT, Barros JA. Self-controlled amount of practice benefits learning of a motor skill. Res Q Exerc Sport. 2011;82:474-81.

11. Janelle C, Barba D, Frehlich S, Tennant L, Cauraugh J. Maximizing performance effectiveness through videotape replay and a self-controlled learning environment. Res Q Exerc Sport. 1997;68:269-79.

12. Chiviacowsky S, Wulf G. Self-controlled feedback: does it enhance learning because performers get feedback when they need it? Res Q Exerc Sport. 2002;73:408-15.

13. Patterson JT, Carter M. Learner regulated knowledge of results during the acquisition of multiple timing goals. Hum Mov Sci. 2010;29:214-27. 
14. Patterson JT, Carter M, Sanli E. Decreasing the proportion of self-control trials during the acquisition period does not compromise the learning advantages in a self-controlled context. Res Q Exerc Sport. 2011;82:624-33.

15. Chiviacowsky S, Wulf G, Medeiros F, Kaefer A, Tani G. Learning benefits of self-controlled knowledge of results in 10-years-old children. Res Q Exerc Sport. 2008;79:405-10.

16. Chiviacowsky S, Neves C, Locatelli L, Oliveira C. Aprendizagem motora em crianças: efeitos da frequência autocontrolada de conhecimento de resultado. Rev Bras Ciênc Esporte. 2005;26:177-90.

17. Róbeka N, Rankélova M. Escola de campeãs. São Paulo: Ícone; 1991.

18. FIG. Federação Internacional de Ginástica. Código de Pontuação de Ginástica Rítmica, 2009-2012.

19. Watkins D. Students' perceptions of factors influencing tertiary learning. High Educ Res Dev. 1984;3:33-50.

20. McCombs ML. Self-regulated learning and achievement: a phenomenological view. In: Zimmerman BJ, Schunk DH. Self-regulated learning and academic achievement theory, research, and practice: progress in cognitive development research. New York: Springer-Verlag; 1989. p.51-82.

21. Chen D, Singer RN. Self-regulation and cognitive strategies in sport participation. Int J Sport Psychol. 1992;23:277-300.

22. Kirschenbaum DS. Self-regulation and sport psychology: nurturing an emerging symbiosis. J Sport Psychol. 1984;6:159-83.

23. Bandura A. Perceived self-efficacy in cognitive development and functioning. Educ Psychol. 1993;28:117-48.

24. Boekaerts M. Self-regulated learning at the junction of cognition and motivation. Eur Psychol. 1996;1:100-12.

25. Chiviacowsky S, Wulf G. Self-controlled feedback is effective if it is based on the learner's performance. Res Q Exerc Sport. 2005;76:42-8.

26. Chiviacowsky S, Wulf G. Feedback after good trials enhances learning. Res Q Exerc Sport. 2007;78:40-7.

27. Chiviacowsky S, Wulf G, Wally R, Borges T. Knowledge of results after good trials enhances learning in the elderly. Res Q Exerc Sport. 2009;80:663-8.

28. Badami R, Vaezmousavi M, Wulf G, Namazizadeh M. Feedback after good trials enhances intrinsic motivation. Res Q Exerc Sport. 2011;82:360-4.

29. Saemi E, Wulf G, Varzaneh AG, Zarghami M. Feedback after good versus poor trials enhances learning in children. Rev Bras Educ Fís Esporte. 2011;25:671-9.

\begin{tabular}{r|l} 
ENDEREÇo & \\
Suzete Chiviacowsky & Recebido para publicação: 31/o8/2012 \\
Universidade Federal de Pelotas & Revisado: 17/02/2013 \\
R. Luís de Camões, 625 - Tablada & Aceito: 05/07/2013 \\
96055-630 - Pelotas - RS - BRASIL & \\
e-mail: schivi@terra.com.br &
\end{tabular}

